\title{
ROLLING OF A SINGLE-CAVITY HYPERBOLOID OF ROTATION ON A HELICOID ON WHICH IT BENDS
}

\author{
Tetiana Kresan - Serhii Pylypaka - Zynovii Ruzhylo - Ivan Rogovskii - Oleksandra Trokhaniak*
}

National University of Life and Environmental Sciences of Ukraine

\begin{tabular}{|c|c|}
\hline ARTICLE INFO & Abstract: \\
\hline $\begin{array}{l}\text { Article history: } \\
\text { Received: } 16.12 .2019 . \\
\text { Received in revised form: } 3.3 .2020 \text {. } \\
\text { Accepted: } 9.3 .2020 \text {. }\end{array}$ & $\begin{array}{l}\text { The bending of a single-cavity hyperboloid with rotation while } \\
\text { maintaining the rectilinear generator is considered. The resulting } \\
\text { bending surfaces are a plural of open skew helicoids, including } \\
\text { partial cases of oblique closed and open ordinary helicoid s. The }\end{array}$ \\
\hline $\begin{array}{l}\text { Keywords: } \\
\text { Single-cavity hyperboloid of rotation } \\
\text { Bending } \\
\text { Helicoids } \\
\text { Linear contact } \\
\text { Rolling } \\
\text { Coefficients of the first quadratic form } \\
\text { DOI: https://doi.org/10.30765/er.1563 }\end{array}$ & $\begin{array}{l}\text { parametric equations for the continuous bending of these surfaces } \\
\text { are established by changing the angle between the straight line } \\
\text { and its axis. The possibility of pure unrolling of a hyperboloid } \\
\text { along a helicoid from the set of its bends with linear contact along } \\
\text { a common rectilinear generator of both surfaces is shown. Using } \\
\text { the obtained equations, the surfaces are constructed and the } \\
\text { images of hyperboloid and helicoid with the common rectilinear } \\
\text { generator of their contact are shown. }\end{array}$ \\
\hline
\end{tabular}

\section{Introduction}

Hyperboloids serve as axoids in the design of gears between passing axes. On this basis, such transmissions are called hyperboloid [1]. When one hyperboloid rolls onto another, their contact occurs in a common rectilinear generator, which is the instantaneous axis of rotation and sliding, i.e., the instantaneous screw axis. Sliding along the line of contact causes increased surface wear. In [2, p. 519] it is stated that the linear surfaces can roll one after the other without sliding when they are bent on each other. It is known from differential geometry that the surface of rotation can be bent to form a screw surface. The article deals with such a bending of a rotational hyperboloid, where the rectilinear generators remain straight and the parallels are transformed into screw lines. Analysis of recent research. The unrolling of single hyperboloids can serve as a basis for the formation of kinematic surfaces. This approach is widely used in papers [3-7]. They consider cases where one axis body is rolled inward and outward by another. Traditional research focuses on considering rotational hyperboloids with a cavity in the roll of axles in the design of gears [8-11]. The design of curves for other kinematic parameters is considered in [12]. In this paper, we are concerned with bending a single-cavity hyperboloid into a helicoid and investigating the possibility of rolling these surfaces individually.

\section{Experimental investigation}

A single-cavity hyperboloid of rotation can be obtained by rotating a rectilinear generator around a passing vertical axis. Based on this method of formation, we write the parametric equations of a hyperboloid:

$$
\begin{aligned}
& X=r \cos \alpha-u \cos \beta \sin \alpha ; \\
& Y=r \sin \alpha+u \cos \beta \cos \alpha ; \\
& Z=u \sin \beta,
\end{aligned}
$$

where $\alpha$ - the angle of rotation of the point around the axis OZ, $\mathrm{u}$ - the length of the rectilinear generator independent variables of surface; $r$ - radius of the smallest parallel (striction line), $\beta$ - the angle at which all

\footnotetext{
${ }^{*}$ Corresponding author.

E-mail address: klendii_o@ukr.net.
} 
rectilinear generators of hyperboloids intersect the striction line - constant magnitudes. On Fig. 1 by equations (1), a single-cavity hyperboloid of rotation with a vertical axis, which has a common contact line with the same hyperboloid, is constructed. To ensure this contact, the hyperboloid is rotated about the axis $O Y$ by an angle $\theta=\pi-2 \beta$ and is shifted along the axis $O X$ by the radius $r$. Rolling of one surface over the other occurs in such a way that the parallels of one surface are rolled over the corresponding parallels of the other, but sliding occurs along the common tangent rectilinear generators. All rectilinear generators of hyperboloid are located in the rectifying plane of the striction line, which is the smallest parallel - the circle of radius $r$. We will bend the surface in such a way that the rectilinear generators of surfaces are preserved and also located in the rectifying plane of the curve, into which the striction line will be transformed. In this case, the hyperboloid bends to the helicoid. The condition of such bending is given in [13, 14]:

$$
\sigma \sin \beta-k \cos \beta=\sigma_{b} \sin \beta-k_{b} \cos \beta
$$

where $k$ and $\sigma$ - the curvature and torsion of the original helical line, respectively, into which the striction line of the hyperboloid will be transformed; $k_{b}$ and $\sigma_{b}$ - the curvature and torsion of the transformed screw line, which change as the surface bends; $\beta$ - constant angle between surface lines (transformed screw line and rectilinear generator).

The parametric equations of a screw line with the angle $\gamma$ of its rise will be written:

$$
x=a \cos \alpha ; \quad y=a \sin \alpha ; \quad z=a \alpha \operatorname{tg} \gamma
$$

where $a$-the radius of the cylinder on which the screw line is located - constant; $\alpha$ - the angle of rotation of the point of the screw line around the $\mathrm{OZ}$ axis is variable.

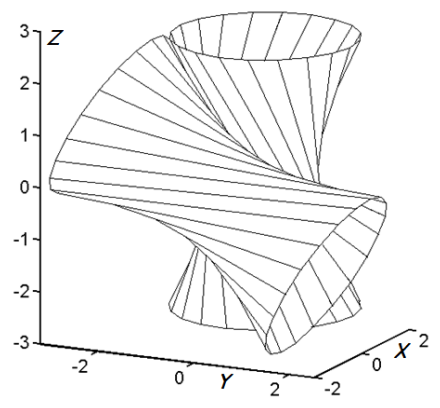

a)

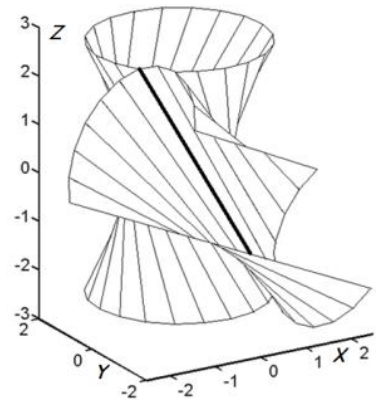

b)

Figure 1. Congruent single-cavity hyperboloids of rotation with common tangent rectilinear generator: a) visual image $b$ ) the common tangent line is depicted thickened.

By known equations we find the curvature $k$ and torsion $\sigma$ of the screw line (3):

$$
k=\frac{\cos ^{2} \gamma}{a} ; \quad \sigma=\frac{\sin \gamma \cos \gamma}{a}
$$

We will transform the screw line (3) by changing its angle of rise $\gamma$. To do this, multiply this angle by a constant value $p: \gamma_{b}=p \cdot \gamma$. At $p=1$ we will have the original screw line, at $p=0$ - the circle, that is, the striction line, in which will turn the screw line when bending the helicoid.

Substitute a new value of the angle $\gamma_{b}=p \cdot \gamma$ into expressions (4) and marking a new value of constant $a$ through $a_{b}$, we write:

$$
k_{b}=\frac{\cos ^{2} p \gamma}{a_{b}} ; \quad \sigma_{b}=\frac{\sin p \gamma \cos p \gamma}{a_{b}}
$$


Expressions (4) describe the curvature and torsion of the original screw line before bending the surface, and expressions (5) after bending. The constant $p$ is the bending parameter. Substitute expressions (4) and (5) into equation (2) and after solving it with respect to $a_{b}$ we obtain:

$$
a_{b}=\frac{a \cos p \gamma \cos (p \gamma+\beta)}{\cos \beta \cos (\gamma+\beta)}
$$

Substitute in the parametric equations of the screw line (3) a new value of constant $a_{b}$ instead of $a$ and a new value of the angle $\gamma_{b}=p \cdot \gamma$ instead of $\gamma$ and obtain:

$$
\begin{aligned}
& x_{b}=\frac{a \cos p \gamma \cos (p \gamma+\beta)}{\cos \gamma \cos (\gamma+\beta)} \cos \alpha_{b} ; \\
& y_{b}=\frac{a \cos p \gamma \cos (p \gamma+\beta)}{\cos \gamma \cos (\gamma+\beta)} \sin \alpha_{b} ; \\
& z_{b}=\frac{a \sin p \gamma \cos (p \gamma+\beta)}{\cos \gamma \cos (\gamma+\beta)} \alpha_{b} .
\end{aligned}
$$

In equations of the transformed screw line (7), the independent variable is denoted by $\alpha_{b}$. Its relation to the independent variable $\alpha$ of the original screw line (3) is found because of the equality of the length of the arc $s$, which does not change when the surface is bent. By known equations we find the length of the arc of the screw lines (3) and (7):

$$
s=\frac{a}{\cos \gamma} \alpha ; \quad s_{b}=\frac{\cos (\gamma+\beta)}{\cos (p \gamma+\beta)} \alpha_{b} .
$$

Equate expressions (8) with each other and solve with respect to $\alpha_{b}$ :

$$
\alpha_{b}=\frac{\cos (\gamma+\beta)}{\cos (p \gamma+\beta)} \alpha \text {. }
$$

Finally, let us proceed in expression (9) to the length of the arc $s$ of the transformed screw line. Since it does not change during bending and is equal to the length of the arc of the original curve, then from the first expression (8) we find: $\alpha=s \cdot \cos \gamma / a$. Substituting this expression in (9) we find a new dependence $\alpha_{b}=\alpha_{b}(s)$. Then we finally write the parametric equations of the helical line (7) as a function of the arc length $s$, the angle of rise $\gamma$ of which will depend on the value of the parameter $p$ :

$$
\begin{aligned}
& x_{b}=\frac{a \cos p \gamma \cos (p \gamma+\beta)}{\cos \gamma \cos (\gamma+\beta)} \cos \left(\frac{\cos \gamma \cos (\gamma+\beta)}{a \cos (p \gamma+\beta)} s\right) \\
& y_{b}=\frac{a \cos p \gamma \cos (p \gamma+\beta)}{\cos \gamma \cos (\gamma+\beta)} \sin \left(\frac{\cos \gamma \cos (\gamma+\beta)}{a \cos (p \gamma+\beta)} s\right) ; \\
& z_{b}=s \sin p \gamma .
\end{aligned}
$$

The striction line of the hyperboloid was transformed into a screw line (10). The rectilinear generator passes through each of its points, which intersects the helical line at an angle $\beta$. If we find the coordinates $N_{x}$, $N_{y}, N_{z}$ of a single directional vector $\bar{N}$ of a rectilinear generator, then we can write the parametric equations of helicoid in the form:

$$
X=x_{b}+u N_{x} ; \quad Y=y_{b}+u N_{y} ; \quad Z=z_{b}+u N_{z}
$$


where $x_{b}, y_{b}, z_{b}$ - parametric equations of the screw line (10); $u$-length of rectilinear generator is the second independent variable of the surface.

The angle $\beta$ during bending of the surface remains constant, so after its bending and the corresponding transformation of the screw line, the directing vector $\bar{N}$ of the rectilinear generator will be one of the cone generator s forming the constant angle $\beta$ with its axis.

In Figure 2, a in the system of the accompanying three-edge of Frenet $\bar{\tau}, \bar{n}, \bar{b}$ of screw line 1 shows the vector $\bar{N}$. The horizontal projection of the fragment of the screw line is indicated by the number 2 . The angle between the tangent to the screw line and its horizontal projection (the angle of rising of the screw line) is indicated by the letter $\gamma$.

According to the bending condition, the rectilinear generator of the surface is located in the rectifying plane of the striction line of the hyperboloid and remains there when the surface is bent and the striction line is converted into a screw one.

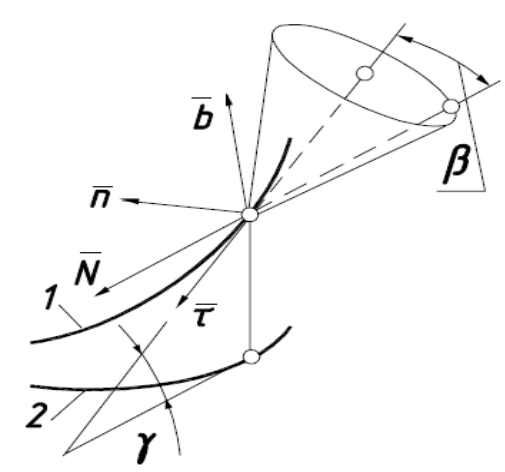

a)

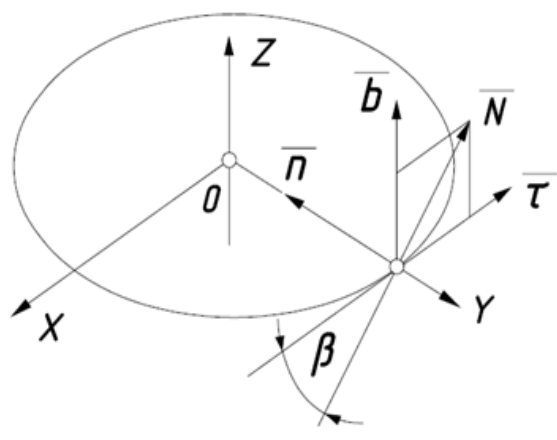

b)

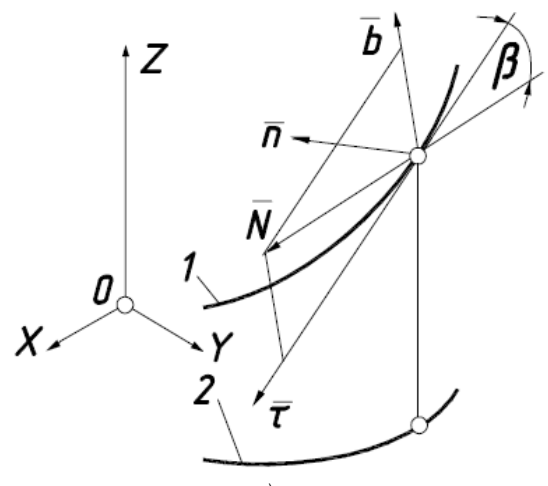

c)

Figure 2. Graphic illustrations for determining the single directional vector of a rectilinear generator of the helicoid when it bending:

a) directing vector is one of the rectilinear generators of the cone whose axis is tangent to the screw line;

b) location of the vector in the rectifying plane of the striction line of the hyperboloid;

c) location of the vector in the rectifying plane of the screw line of the helicoid.

The projections of the directional unit vector $\bar{N}$ onto the three-edge of Frenet, regardless of their location on the striction line, will be recorded in the projections on the triangular orts (Figure 2, b):

$$
N_{\tau}=\cos \beta ; \quad N_{n}=0 ; \quad N_{b}=\sin \beta .
$$


The same angle $\beta$ will be in the rectifying plane of the screw line (10) between the unit tangent vector and the directing vector of the rectilinear generator of the helicoid. The transition from the coordinates of the vector (12) in the moving trihedral to its coordinates in the fixed OXYZ system is made by known transfer equation:

$$
\begin{aligned}
& N_{x}=N_{\tau} \cos \alpha_{\tau}+N_{n} \cos \alpha_{n}+N_{b} \cos \alpha_{b} ; \\
& N_{y}=N_{\tau} \cos \beta_{\tau}+N_{n} \cos \beta_{n}+N_{b} \cos \beta_{b} ; \\
& N_{z}=N_{\tau} \cos \gamma_{\tau}+N_{n} \cos \gamma_{n}+N_{b} \cos \gamma_{b},
\end{aligned}
$$

where $\alpha_{\tau}, \beta_{\tau}, \gamma_{\tau}$ are angles formed by the unit tangent vector with the axes OX, OY, and OZ of the fixed coordinate system; $\alpha_{n}, \beta_{n}, \gamma_{n}$ and $\alpha_{b}, \beta_{b}, \gamma_{b}$ - are the angles between the axis of the fixed coordinate system and the unit normal vector $\bar{n}$ and unit binormal vector $\bar{b}$ of the trihedral, respectively.

The cosines of these angles are determined by the first and second derivatives of the screw line equations (10):

$$
\begin{aligned}
& \cos \alpha_{\tau}=\frac{x_{b}^{\prime}}{\sqrt{x_{b}^{\prime 2}+y_{b}^{\prime 2}+z_{b}^{\prime 2}}} ; \\
& \cos \alpha_{b}=\frac{A}{\sqrt{A^{2}+B^{2}+C^{2}}} ; \\
& \cos \beta_{\tau}=\frac{y_{b}^{\prime}}{\sqrt{x_{b}^{\prime 2}+y_{b}^{\prime 2}+z_{b}^{\prime 2}}} ; \\
& \cos \beta_{b}=\frac{B}{\sqrt{A^{2}+B^{2}+C^{2}}} ; \\
& \cos \gamma_{\tau}=\frac{z_{A}^{\prime}}{\sqrt{x_{b}^{\prime 2}+y_{b}^{\prime 2}+z_{b}^{\prime 2}}} ; \\
& \cos \gamma_{b}=\frac{C}{\sqrt{A^{2}+B^{2}+C^{2}}}
\end{aligned}
$$

where $A=y_{b}^{\prime} z_{b}^{\prime \prime}-y_{b}^{\prime \prime} z_{b}^{\prime} ; B=z_{b}^{\prime} x_{b}^{\prime \prime}-z_{b}^{\prime \prime} x_{b}^{\prime} ; C=x_{b}^{\prime} y_{b}^{\prime \prime}-x_{b}^{\prime \prime} y_{b}^{\prime}$. The expressions for the cosines of the angles $\alpha_{n}$, $\beta_{n}, \gamma_{n}$ are not given since $N_{n}=0$ and they are not required for equations (13).

Substitute the found cosines (14) and expressions (12) into equations (13) and after simplifications we obtain:

$$
\begin{aligned}
& N_{x}=-\cos (p \gamma+\beta) \sin \left(\frac{\cos \gamma \cos (\gamma+\beta)}{a \cos (p \gamma+\beta)} s\right) \\
& N_{y}=\cos (p \gamma+\beta) \cos \left(\frac{\cos \gamma \cos (\gamma+\beta)}{a \cos (p \gamma+\beta)} s\right) \\
& N_{z}=\sin (p \gamma+\beta) .
\end{aligned}
$$

Substitute expressions (10) and (15) in (11) and obtain the parametric equations of the surface, which is the result of the bending of a single-cavity hyperboloid of rotation: 


$$
\begin{gathered}
X=\frac{a \cos p \gamma \cos (p \gamma+\beta)}{\cos \gamma \cos (\gamma+\beta)} \cos \left(\frac{\cos \gamma \cos (\gamma+\beta)}{a \cos (p \gamma+\beta)} s\right)- \\
-u \cos (p \gamma+\beta) \sin \left(\frac{\cos \gamma \cos (\gamma+\beta)}{a \cos (p \gamma+\beta)} s\right) \\
Y=\frac{a \cos p \gamma \cos (p \gamma+\beta)}{\cos \gamma \cos (\gamma+\beta)} \sin \left(\frac{\cos \gamma \cos (\gamma+\beta)}{a \cos (p \gamma+\beta)} s\right)+ \\
+u \cos (p \gamma+\beta) \cos \left(\frac{\cos \gamma \cos (\gamma+\beta)}{a \cos (p \gamma+\beta)} s\right) \\
Z=s \sin p \gamma+u \sin (p \gamma+\beta) .
\end{gathered}
$$

At $p=0$ equation (16) describes a single-cavity hyperboloid of rotation, at $p=\pi /(2 \gamma)-$ closed skew helicoid, at intermediate values of $p$-open skew helicoids.

\section{Results and discussion}

To verify the accuracy of the obtained parametric equations (16) of the bending of the surface, we find the coefficients of the first quadratic form; they are determined by partial derivatives:

$$
\begin{aligned}
& E=\left(\frac{\partial X}{\partial u}\right)^{2}+\left(\frac{\partial Y}{\partial u}\right)^{2}+\left(\frac{\partial Z}{\partial u}\right)^{2}=1 \\
& F=\frac{\partial X}{\partial u} \cdot \frac{\partial X}{\partial s}+\frac{\partial Y}{\partial u} \cdot \frac{\partial Y}{\partial s}+\frac{\partial Z}{\partial u} \cdot \frac{\partial Z}{\partial s}=\cos \beta \\
& G=\left(\frac{\partial X}{\partial s}\right)^{2}+\left(\frac{\partial Y}{\partial s}\right)^{2}+\left(\frac{\partial Z}{\partial s}\right)^{2}= \\
& =1+\frac{u^{2}}{a^{2}} \cos ^{2} \gamma \cos ^{2}(\gamma+\beta) .
\end{aligned}
$$

Coefficient expressions do not include parameter $p$. From its value the shape of the surface changes, but the linear element, which is determined by the coefficients (17), remains unchanged. This is confirmation that equations (16) are indeed surface bending equations. On Figure 3, a single-cavity hyperboloid of rotation according to equations (16) is constructed at $\mathrm{p}=0$ and bounded by the striction line (with the parameter $\mathrm{u}$ changing from zero to a given length of a rectilinear generator). According to the same equations, a helicoid was constructed at $p=0.5$, and it was rotated and transferred so that the rectilinear generators coincide (the common line of contact on the figures is shown in thick).

The angle of rotation and the magnitude of the transfer are determined as follows. For $s=$ const (for example, $s=0$ ) and $u=0$, corresponding points will be found on the striction line of the hyperboloid and on screw line of the helicoid. The coordinates of these points combine them. Then the angle $\theta$ between rectilinear generators by equations (15) for a hyperboloid (at $p=0$ ) and a helicoid (at $p=0.5$ ) is determined. For accepted values of constants: $a=1, \beta=45^{\circ}, \gamma=-45^{\circ}$ angle $\theta=22.5^{\circ}$. In the initial position, axes of surfaces are vertical. Turning on the angle $\theta$ to one side or the opposite side, one of the surfaces remains with the vertical axis and the other returns to the combination with the rectilinear generators.

When you roll a hyperboloid along the helicoid, the corresponding rectilinear generators are aligned in sequence and the circles will be rolling along screw lines. Since the bending surfaces do not change the length of the lines, the angles between them, or the surfaces, the rolling occurs without sliding.

In a special case, when a surface is curved, there may be an open ordinary spiral whose rectilinear generators are parallel to the horizontal plane of projection. In this case, the vertical component $\mathrm{Nz}$ of the direction vector (15) must be zero. From here we find: $p=-\beta / \gamma$. For the angles $\beta=45^{\circ}, \gamma=-45^{\circ}$ we obtain $p=1$. To see that the generators $\mathrm{s}$ of the helicoid are parallel to the horizontal plane OXY, we constructed the frontal projections of an open ordinary helicoid with vertical axis (Figure 4). The rectilinear generator of the 
hyperboloid in contact with the helicoid is represented by a thickened line. For clarity, a fragment of a cylindrical surface in the form of a wave is shown in Figure 4, a.

In Figure 4, c the rectilinear generators of the hyperbolic paraboloid are elongated on both sides of the friction line. Accordingly, the generators on the helicoid are also elongated. Since the helicoid is open, it has an open region where the cylindrical shaft is located.

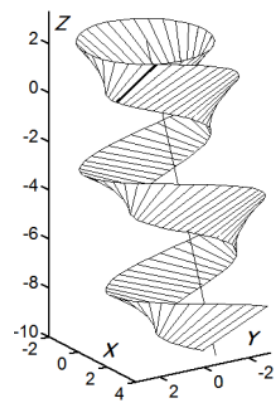

a)

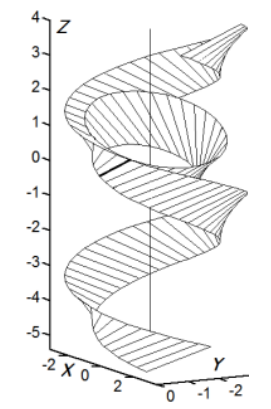

b)

Figure 3. A single-cavity hyperboloid of rotation constructed by equations (16) at $p=0$ and the helicoid constructed by the same equations at $p=0.5$, touch each other along a common rectilinear generator:

a) axis of the hyperboloid is vertical, axis of the helicoid is inclined;

b) axis of the hyperboloid is inclined, axis of the helicoid is vertical

For illustration this case is shown in axonometry on Figure 5, a. From it can be seen the curve of indirection of the theorem of rectilinear generators, which resembles in form a hyperbola. Obviously, such a curve is an axial section of the surface. When the surface is curved by stretching it along the axis, that is, when the value of the parameter $p$ increases, the curve of the axial section is transformed until it becomes a straight line - the axis of the closed helicoid (Figure 5, c).

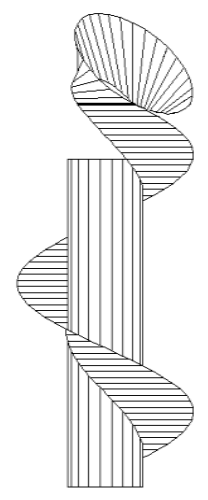

a)

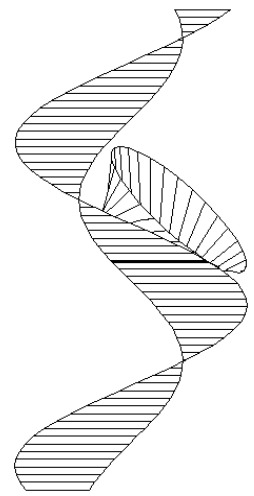

b)

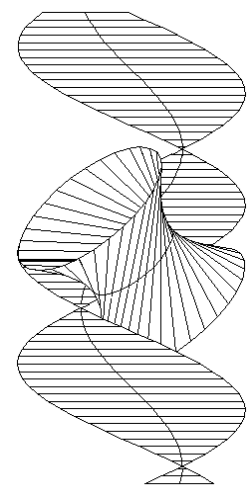

c)

Figure 4. Frontal projection of open ordinary helicoid s with tangent single-cavity hyperboloids of rotation:

a) open ordinary helicoid with fragment of coaxial shaft;

b) helicoid and hyperboloid, their length of the generator $u$ varies within $u=0 \ldots 2$;

c) helicoid and hyperboloid, their length of the generator $u$ varies within $u=-2 \ldots 2$. 


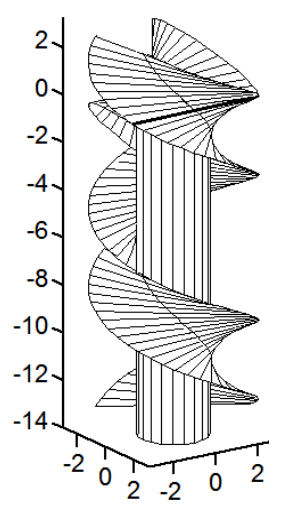

a)

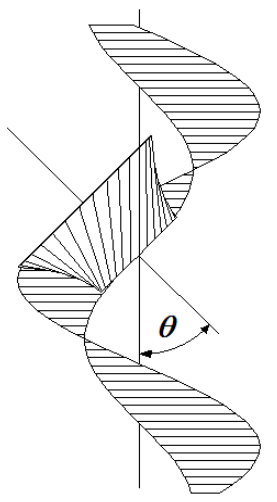

b)

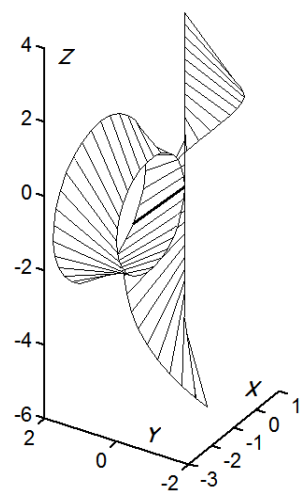

c)

Figure 5. A single-cavity hyperboloid of rotation constructed by equations (16) at $p=0$ and the helicoid constructed by the same equations at $p=0.5$, touch each other along a common rectilinear generator:

a) axis of the hyperboloid is vertical, axis of the helicoid is inclined;

b) axis of the hyperboloid is inclined, axis of the helicoid is vertical

On Figure 5, b, a frontal projection of a hyperboloid and a helicoid is shown such that the angle between their axes is projected in the natural value. For this case $=45^{\circ}$. When the line of elongation of the hyperboloid changes to a straight line - the axis of the closed oblique helicoid in this case the angle $\theta=90^{\circ}$ (Figure 5, b). The distance between the axes in this case is maximum - it is equal to the radius of the friction line. In the other cases it is equal to the difference between the point of contact of the surfaces on the friction line and their axes. If you bend the surface by compression, the distance and the angle between the axes become smaller. When the helicoid becomes a rotational hyperboloid, the distance between the axes and the angle between them become zero.

For a certain value of the bending parameter $p$, the angle is constant (for example $\theta=45^{\circ}$ for $p=1$, Figure $5, \mathrm{~b}$ ). If the axis of rotation of the hyperboloid is stationary and the hyperboloid is rotated about this axis, the helicoid is forced to rotate about its own axis while sliding along this axis to ensure contact between the surfaces. A hyperboloid-helicoid pair can thus be an alternative to pairs of screws that convert rotational motion into translational motion. When such a pair is used, there is no sliding friction in the contact zone of the surfaces.

\section{Conclusion}

A single-cavity hyperboloid can be bent into a helicoid by stretching its extensor line into a helical line. It is possible to obtain a series of open oblique helicoids, open ordinary helicoids, and closed oblique helicoids. On each of these surfaces the original surface can roll - the hyperboloid of rotation. When rolling in the area of straight contact, there is no sliding friction, which significantly reduces the wear of the surfaces. The distance and angle between the axes for a given pair of surfaces are constant. Such a pair can convert the rotational motion of the hyperboloid into a screw motion of the helicoid, that is, it can be an alternative to pairs of screws.

\section{References}

[1] Levitskaia O.N., Levitskyi N.I.: The course of the theory of mechanisms and machines, High School, Moskow / Russia, 1985.

[2] Shulikovskyi V.I.: Classical differential geometry in tensor exposition, Fizmatgiz, Moskow / Russia, 1963.

[3] Rachkovskaya G.S., Kharabaev Yu.N.: Kinematic ruled surfaces based on the complex movement of one axoid over another (Single-cavity hyperboloids of rotation as fixed and moving axoids), Structural Mechanics of Engineering Structures and Facilities, 3 (2014). 23-30. 
[4] Rachkovskaya G.S.: Mathematical modeling of kinematic surfaces based on a single-cavity hyperboloid of rotation as a fixed and moving axoids, Electronic scientific journal "Engineering Bulletin of the Don", 1 (2013).

[5] Rachkovskaya, G.S., Kharabayev, Yu.N., Rachkovskaya N.S.: Kinematic ruled surfaces (one-sheet hyperboloid of revolution as fixed and moving axoids), Proceedings of the 13 th International Conference on Geometry and Graphics, Dresden / Germany, 2008, 190-191.

[6] Rachkovskaya, G.S., Kharabayev, Yu.N.: Geometric modeling and computer graphics of kinematic ruled surfaces on the base of complex moving one axoid along another (one-sheet hyperboloid of revolution as fixed and moving axoids), Proceedings of the 17-th International Conference in Central Europe on Computer Graphics, Visualization and Computer Vision, Plzen / Czech Republic, 2009, 3134.

[7] Rachkovskaya, G.S., Kharabayev, Yu.N.: Geometric model of kinematic surfaces on the base of onesheet hyperboloidal surfaces of revolution (one axoid is located in the interior of another axoid), Proceedings of the 14th International Conference on Geometry and Graphics, Kyoto / Japan, 2010, 320321.

[8] Shcherbakov N.R., Shchegoleva A.A.: Touching of single-cavity hyperboloids of rotation as hypoid transmission axoids, Mathematics and Mechanics. Bulletin of Tomsk State University, 47 (2017), 3742.

[9] Radzevych S.P.: Vector representation of gear pairs. Part 1, Theory of Mechanisms and Machines, 6 (2008), 6, 74-81.

[10] Radzevych S.P.: Vector representation of gear pairs. Part 2, Theory of Mechanisms and Machines, 1 (2009), 7, 17-26.

[11] Zhang G., Xiang Y., Guo H., Nie Y.: Structural reliability and its sensitivity analysis based on the saddlepoint approximation-line sampling method by dichotomy of golden section, Engineering Review, Rijeka / Croatia, 39 (2019), 1, 11-20.

[12] Bulgakov V., Pilipaka S., Adamchuk V., Olt J.: Theory of motion of a material point along a plane curve with a constant pressure and velocity, Agronomy Research, Estonian Agricultural University, 12 (2014), 3, 937-948.

[13] Pylypaka S.F.: Continuous flexion of the skew helicoid into a single-cavity hyperboloid of rotation, Applied Geometry and Engineering Graphics, Kyiv / Ukraine, 61 (1996), 140-144.

[14] Pylypaka, S.F., Klendii, M.B., Nesvidomin, V.M., Trokhaniak, V.I.: Particle motion over the edge of an inclined plane that performs axial movement in a vertical limiting cylinder, Acta Polytechnica / Czech Republic, 59 (2019), 1, 67-76. 